\title{
PIONIC DISINTEGRATION OF THE DEUTERON
}

\author{
M. BRACK ${ }^{\dagger}$ \\ Department of Physics, State University of New York, Stony Brook, New York 11794 \\ D. O. RISKA ${ }^{\dagger+}$ \\ Department of Physics, Michigan State University, East Lansing, Michigan 48824 \\ and \\ W. WEISE ${ }^{\dagger+t}$ \\ Fachbereich Physik, Universität Regensburg, Regensburg, W. Germany
}

Received 11 March 1977

\begin{abstract}
Abatract: Pionic disintegration of the deuteron between threshold and the 3,3 resonance region is described by a model containing one- and two-body absorption. The two-body absorption mechanism is due to pion and $\rho$-meson rescattering calculated from phenomenological Lagrangians. The role of the $\rho$-meson is crucial in reducing the cross section due to pion exchange. The role of the mass distribution of the $\rho$-meson, hadronic form factors and final state interactions are investigated. Good agreement with empirical results for the total crose section is obtained with a set of currently accepted values for the meson-nucleon coupling constants and the $\rho$-mass distribution.
\end{abstract}

\section{Introduction}

Pionic disintegration of the deuteron, $\pi^{+} \mathrm{d} \rightarrow \mathrm{pp}$, and the inverse reaction are of fundamental interest as they provide the simplest examples of nuclear pion absorption and production. These reactions have therefore drawn considerable attention in the literature ${ }^{1}$ ) and have been analyzed, but not very well understood, in terms of simple semi-phenomenological models. Experimental results for the cross sections extend from threshold to pion energies of some three times the pion mass ${ }^{1.2}$ ).

Near threshold the absorption process is dominated by S-wave rescattering ${ }^{3}$ ). At larger pion momenta $\mathbf{P}$-wave rescattering through an intermediate state containing a $\Delta_{33}$ resonance governs the total cross section ${ }^{4,5}$ ), an effect evidenced by a broad peak in the cross section.

The general interest in the $\pi^{+} d \rightarrow p p$ reaction has a number of motivations. The foremost of these is the realization that unless this reaction is understood there is little reason to expect that pion capture and production for more complex nuclei

+ Supported in part by USERDA contract E(11-1)-3Q01. Present address: Inatitut Laue-Langevin, Grenoble, France.

it Supported in part by the National Science Foundation.

tt' Supported in part by USERDA contract E(11-1)-3001. 
can be understood either. Another motivation is the study of the rescattering mechanisms and the question of whether in that context something might be learned about the off-shell pion-nucleon interaction.

Goplen, Gibbs and Lomon ${ }^{6}$ ) have recently pursued this point. Their description of pion rescattering was based on the use of a phenomenological $\pi \pi \mathrm{NN}$ Hamiltonian, the on-shell properties of which where adjusted so as to reproduce free $\pi \mathrm{N}$ scattering. The off-shell characteristics were described by a phenomenological cut-off factor at the rescattering vertex. To obtain agreement with the empirical total cross-section values Goplen, Gibbs and Lomon found that the cut-off factor needed to have rather long range (the mass scale being 2.7 times the pion mass). Now it seems impossible to find the dynamical origin for that long range mass scale in the pion nucleon amplitude. A natural explanation of the result is that the long range form factor simply mocks up the effects of neglected short range rescattering mechanisms rather than giving a realistic description of the off-shell properties of the $\pi \mathrm{N}$ interaction. The phenomenological character of the result of ref. ${ }^{6}$ ) is illustrated by the later result of Gibbs, Gibson and Stephenson ${ }^{7}$ ) who found that the range of the form factor becomes considerably shorter upon addition of another cut-off factor associated with the final pion absorption vertex (the mass scale changes to 4.3 pion masses). We interpret this result to indicate that the original form factor mass scale was unrelated to the off-shell $\pi \mathbf{N}$ interaction.

In a recent publication ${ }^{8}$ ) we showed that $\rho$-meson rescattering following the excitation of an intermediate $\Delta_{33}$ resonance cancels a sufficiently large fraction of the pion exchange contribution to the total cross section so that there remains little need for the introduction of form factors of any appreciable range. This result demonstrates an obvious need to take into account properly short range exchange effects in addition to pion exchange.

The purpose of the present paper is to present the model given in ref. $\left.{ }^{8}\right)$ in detail and to extend it in several directions. We consider the effect of replacing the sharp resonance approximation for the $\rho$-meson exchange mechanism by the mass distribution appropriate for the interacting isospin-1 P-wave $\pi \pi$ state. The sensitivity of the calculated cross sections to hadronic form factors associated with the $\pi$ - and $\rho$-vertices is studied and we reach the conclusion that the form factor cut-off mass scale must be considerably larger than $1 \mathrm{GeV} / c^{2}$.

The paper is organized in the following way. After a short review of the basic cross-section formulae in sect. 2 , the calculation of P-wave pion absorption is presented in sect. 3. In sect. 4 we review the standard method of calculating S-wave pion absorption. In sect. 5 the effects of the $\rho$-meson mass distribution are discussed. A concluding discussion is given in sect. 6. Some details of the formalism are listed in two appendices at the end of the paper. 
2. The cross section for $\pi^{+} d \rightarrow p p$

We calculate the $\pi$-absorption cross section in the lab system (deuteron rest frame). The absorption process is schematically illustrated in fig. 1 . The box $M$ in the figure

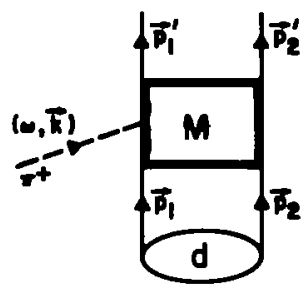

Fig. 1. Kinematic variables for $\pi^{+} d \rightarrow p p$.

represents any of the one- or two-body absorption mechanisms to be discussed in the subsequent chapters.

We introduce the relative and c.m. frame momentum variables

$$
\begin{gathered}
P=p_{1}+p_{2}=0, \quad p=\frac{1}{2}\left(p_{1}-p_{2}\right), \\
P^{\prime}=p_{1}^{\prime}+p_{2}^{\prime}, \quad p^{\prime}=\frac{1}{2}\left(p_{1}^{\prime}-p_{2}^{\prime}\right) .
\end{gathered}
$$

Here $p_{1}$ and $p_{2}$ are the nucleon momenta prior to the absorption of the pion and $\boldsymbol{p}_{1}^{\prime}$ and $\boldsymbol{p}_{2}^{\prime}$ the corresponding posterior nucleon momenta. Assuming that $P^{\prime 2} / M<\omega=\sqrt{k^{2}+\mu^{2}}$ (with $M$ and $\mu$ being the nucleon and pion masses respectively), the cross section can be cast into the form

$$
\mathrm{d} \sigma=\frac{1}{2 k} \frac{\mathrm{d}^{3} P^{\prime}}{(2 \pi)^{3}} \frac{\mathrm{d}^{3} p^{\prime}}{(2 \pi)^{3}}(2 \pi)^{3} \delta\left(P+k-p^{\prime}\right) 2 \pi \delta\left(E_{1}^{\prime}+E_{2}^{\prime}-\omega+B-2 M\right)\left|T_{\mathrm{fi}}\right|^{2} .
$$

Here $B$ is the deuteron binding energy which we forthwith neglect. In eq. (2.2) we use the notation

$$
E_{i}^{\prime}=\left(P_{1}^{\prime 2}+M^{2}\right)^{\frac{1}{2}}
$$

The matrix $T_{\mathrm{fi}}$ in (2.2) contains the operator $\hat{M}$ represented by the box in fig. 1:

$$
T_{\mathrm{fi}}=\left\langle\psi_{\mathrm{f}}\left(\boldsymbol{p}^{\prime}\right)|\hat{M}| \pi(k), \psi_{\mathrm{d}}\right\rangle .
$$

The various contributions to $M$ will be discussed in the following sections.

In the integration over $p^{\prime}$ in (2.2) the energy $\delta$-function picks out the following value for the final relative momentum (neglecting terms of order $k^{2} / 4 M^{2}$ ):

$$
p^{\prime}=\sqrt{M \omega+\frac{1}{4} \mu^{2}} .
$$

The magnitude of $p^{\prime}$ varies between $360 \mathrm{MeV} / c$ at threshold to more than $500 \mathrm{MeV} / c$ in the 3,3 resonance region. As the components in the deuteron wave function 
corresponding to such large momenta have small weight it is clear that most of the momentum transfer must be supplied by $t$-channel mechanisms in $\hat{M}$. Using (2.5) we obtain for the differential and total spin averaged cross sections the expressions

$$
\begin{gathered}
\frac{\mathrm{d} \sigma}{\mathrm{d} \Omega}=\frac{P^{\prime}\left(M+\frac{1}{2} \omega\right)}{k(4 \pi)^{2}} \frac{1}{2 j_{1}+1} \sum_{S_{r}, M_{\mathrm{f}, M_{i}}}\left|T_{\mathrm{f}}\right|^{2}, \\
\sigma=\frac{1}{2} \int \mathrm{d} \Omega \frac{\mathrm{d} \sigma}{\mathrm{d} \Omega} .
\end{gathered}
$$

Here $\Omega$ is the solid angle describing the direction of $p^{\prime}$. The factor $\frac{1}{2}$ in the total cross section takes into account the indistinguishability of the final protons.

For the deuteron wave function $\psi_{d}$ we shall use the solution obtained with the Reid soft-core potential ${ }^{9}$ ). The wave function $\psi_{\mathrm{f}}$ for the pp final state will be described by a plane wave, except for the case of the ${ }^{1} S_{0}$ and ${ }^{1} D_{2}$ waves where we also use scattering solutions obtained with the Reid soft-core potential. We find that the final state interactions are of but little importance in practice. There is a certain need for care in the construction of the operator $M$, when correlated scattering wave functions are employed, lest double counting occur. We return to this point in the next section.

\section{P-wave abeorption mechanisms}

\subsection{IMPULSE APPROXIMATION}

We first review the calculation of the lowest order (impulse approximation) process shown diagrammatically in fig. 2 . The nonrelativistic approximation for the

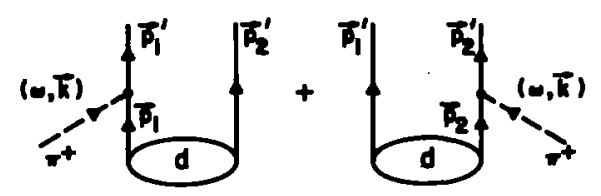

Fig. 2. Diagrammatic representation of single nucleon absorption.

absorption vertex for a pion on a single nucleon is (appendix A)

$$
\left\langle p_{1}^{\prime}\left|\mathscr{L}_{\mathrm{zNN}}\right| \pi^{+}(k), p_{i}\right\rangle=-\frac{f}{\mu} \sigma^{\prime} \cdot\left[k-\frac{E_{l}^{\prime}-E_{l}^{\prime}}{4 M}\left(p_{i}^{\prime}+p_{i}\right)\right] \sqrt{2} \tau_{+}^{i} .
$$

Here $\sigma^{t}$ and $\tau^{t}$ are the spin and isospin Pauli matrices for nucleon $i$ and $f^{2} / 4 \pi=0.08$. The recoil term proportional to $E_{l}^{\prime}-E_{l}$ in (3.1) is of no quantitative importance for $P$-wave absorption and hence we do not consider it in the calculations below. We. return however to a discussion of that term in sect. 4.

The impulse approximation (IA) contribution to the scattering amplitude can be 
expressed as a sum of two single-nucleon operators as

$$
M_{1 \mathrm{~A}}^{+}(k, r)=-\frac{f}{\mu}\left\{\mathrm{e}^{\mathrm{e}+k \cdot r}\left(\sigma^{1} \cdot k\right) \sqrt{2} \tau_{+}^{1}+\mathrm{e}^{-\frac{1}{t} \cdot \boldsymbol{k} \cdot r}\left(\sigma^{2} \cdot k\right) \sqrt{2} \tau_{+}^{2}\right\} .
$$

Here $r=r_{1}-r_{2}$ is the relative position coordinate of the two nucleons. The matrix element of this operator is (2.4)

$$
T_{\mathrm{fi}}=\int \mathrm{d}^{3} r \psi_{\mathrm{f}}^{*}\left(p^{\prime}, r\right) M_{\mathrm{IA}}^{+}(k, r) \psi_{\mathrm{d}}(r)
$$

Evaluation of the cross section (2.6) with the IA amplitude above and using a plane wave to describe $\psi_{\mathrm{f}}$ gives less than $2 \mathrm{mb}$ at all energies and the result explains neither the magnitude nor shape of the observed cross section. Nevertheless one has to include the IA amplitude as it will add coherently to the rescattering amplitudes to be considered below.

The smallness of the impulse approximation cross section does not depend much on whether or not correlated final state wave functions are used. The difference amounts to at most $15 \%$ at large energies and to far less at low energies (see fig. 4 below).

\subsection{PION RESCATTERING}

The dominant process above threshold is the rescattering of a meson following excitation of a $A_{33}$ resonance in the intermediate state, as shown in fig. 3. Presently

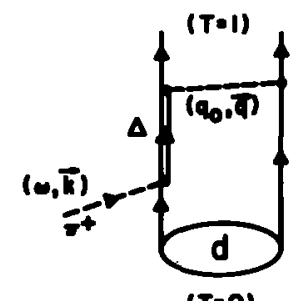

Fig. 3. The P-wave rescattering effect.

we shall consider the rescattered meson to be a pion and consider $\rho$-rescattering in subsect. 3.3. As the rescattered meson is highly off-shell $\left(q_{0}^{2}<q^{2}\right)$ we shall have to discuss the effect of form factors associated with the $\pi \mathrm{NN}$ and $\pi \mathrm{N} \Delta$ vertices appearing in fig. 3. We note that the crossed diagrams in which a meson is exchanged before the absorption of the $\pi^{+}$with accompanying conversion of a $\Delta_{33}$ resonance into a nucleon is forbidden by isospin conservation. Rescattering graphs where the isobar is replaced by a nucleon (i.e. the pion scattering Born terms) should not be included when correlated wave functions are used, as those diagrams are part of the impulse approximation amplitude. 
In the absence of final state correlations the momentum of the rescattered meson is

$$
q=\frac{1}{2} k \mp\left(p^{\prime}-p\right),
$$

where the - or + sign holds for absorption of the pion on nucleon 1 or 2 respectively.

The symmetrized two-body operator which describes the pion rescattering process in fig. 3 has the form

$$
\left\langle 0\left|\hat{M}_{\Delta x}(q)\right| \pi^{+}(k)\right\rangle=\frac{f^{*}}{\mu} \frac{1}{\omega_{\mathrm{R}}-\omega-\frac{1}{2} i \Gamma_{\Delta}}\left\{V_{12}^{\pi}\left(q_{0}, q\right)\left(S_{1}^{+} \cdot k\right) T_{+1}^{+}+V_{21}^{\pi}\left(q_{0}, q\right)\left(S_{2}^{+} \cdot k\right) T_{+2}^{+}\right\},
$$

where $V_{12}^{\pi}$ is the $\Delta \mathrm{N}-\mathrm{NN}$ one-pion-exchange interaction,

$$
V_{12}^{\mathrm{z}}\left(q_{0}, q\right)=\frac{f^{*} f}{\mu^{2}} \frac{S_{1} \cdot q \sigma_{2} \cdot q}{q_{0}^{2}-q^{2}-\mu^{2}} T_{1} \cdot \tau_{2}
$$

Here $S$ and $T$ are transition spin and isospin operators which connect nucleons and isobars ${ }^{10}$ ) and $f^{*}$ is the $\pi N \Delta$ coupling constant for which we use the value $2 f$ as given by the Chew-Low model ${ }^{11,12}$ ). The position of the resonance is given by the expression

$$
\omega_{\mathrm{R}}=M^{*}-M+k^{2} / 2(M+\omega),
$$

if the momentum of the initial nucleon is neglected. The isobar width is

$$
\Gamma_{\Delta}=\frac{2}{3} \frac{f^{*}}{4 \pi} \frac{k^{3}}{\mu^{2}}
$$

in order that the $\mathbf{P}_{33}$ phase shift goes through $90^{\circ}$ at the resonance.

In the absence of final state interactions and with the assumption the nucleons in the deuteron are approximatively at rest, the energy transfer $q_{0}$ is

$$
q_{0} \approx \sqrt{M^{2}+q^{2}}-M
$$

The propagator of the rescattered pion then has the form

$$
D(q)=\left[2 M^{2}\left(1-\sqrt{1+q^{2} / M^{2}}\right)-\mu^{2}\right]^{-1} .
$$

Note that this propagator is non-singular and negative definite.

If the square root in (3.10) is expanded to order $q^{2}$ one obtains the usual static pion propagator

$$
D(q) \approx-1 /\left(q^{2}+\mu^{2}\right)
$$

This approximation preserves the non-singularity and negative definiteness of the correct propagator (3.10). We shall use this approximate form in the calculations.

In ref. $\left.{ }^{6}\right)$, Goplen et al. used the form for the pion propagator which results upon setting $q_{0}=\frac{1}{2} \omega$ rather than using (3.9). That prescription for $q_{0}$ follows if one assumes that the momenta of the final nucleons are equal to the relative momentum in the 
final state. In addition the pion propagator becomes singular for $\omega>2 \mu$, a most undesirable result. Yet the numerical consequences of this singularity are rather small; we have found that replacing the propagator (3.11) with the form used in ref. ${ }^{6}$ ) leads to small increases in the cross section values amounting to less than $10 \%$ over the energy region considered.

Using the static pion propagator (3.11) the configuration space representation of the operator $M$ in (3.5) becomes

$$
M_{\Delta, \pi}^{+}(k, r)=\frac{f^{*}}{\mu} \frac{1}{\omega_{R}-\omega-\frac{1}{2} i \Gamma_{\Delta}}\left\{\mathrm{e}^{+i k \cdot r} V_{12}^{\pi}(r)\left(S_{1}^{+} \cdot k\right) T_{+1}^{+}+\mathrm{e}^{-\frac{1}{t} k \cdot r} V_{21}^{\pi}(r)\left(S_{2}^{+} \cdot k\right) T_{+2}^{+}\right\},
$$

where

$$
V_{12}^{\pi}(r)=\frac{1}{3} \frac{f^{*} f}{4 \pi}\left\{\frac{\mathrm{e}^{-\mu r}}{r} S_{1} \cdot \sigma_{2}+\left(1+\frac{3}{\mu r}+\frac{3}{\mu^{2} r^{2}}\right) \frac{\mathrm{e}^{-\mu r}}{r} S_{12}^{*}(\theta) 1\right\} T_{1} \cdot \tau_{2} .
$$

Here we use the notation

$$
S_{12}^{*}=3 S_{1} \cdot f \sigma_{2} \cdot p-S_{1} \cdot \sigma_{2} .
$$

The calculational details needed to evaluate the matrix elements

$$
T_{\mathrm{f} 1}^{A, x}=\int \mathrm{d}^{3} r \psi_{\mathrm{f}}^{*}\left(p^{\prime}, r\right) M_{\Delta, \mathrm{x}}^{+}(k, r) \psi_{\mathrm{d}}(r)
$$

are given in appendix $B$.

Because the spin and isospin $\frac{3}{2}$ character of the $\Delta_{33}$ resonance there is no contribution from the spin-spin simple Yukawa part of the rescattering operator (3.13) for singlet final states. The whole contribution is from the tensor term. For triplet final states the tensor term is much more important than the spin-spin term. The $\delta$-function interaction associated with the spin-spin term has been omitted as it gives no contribution as long as $\psi_{d}(r=0)=0$.

\subsection{RESCATTERING OF A $\rho$-MESON}

Clearly the exchange of a $\rho$-meson is possible in the rescattering graph in fig. 3. It is expected to be important as the main term in the rescattering operator is a tensor term which emphasizes the short range contributions.

The rescattering of a $\rho$-meson following excitation of a $\Delta$-resonance is ciescribed simply by replacing the one-pion-exchange interaction $V_{12}$ in eq. (3.5) by the corresponding $\rho$-meson-exchange interaction

$$
V_{12}^{\rho}(q)=-\frac{f_{\rho}^{*} f_{\rho}}{m_{\rho}^{2}} \frac{\left(S_{1} \times q\right) \cdot\left(\sigma_{2} \times q\right)}{q^{2}+m_{\rho}^{2}} T_{1} \cdot \tau_{2}
$$

Here the $\rho$-meson is described as a zero width particle with mass $m_{\rho} \approx 5.5 \mu$. In sect. 5 
below we shall treat the $\rho$-meson as an object of distributed mass in the isospin-1 channel of the two-pion-exchange interaction. The $\rho \mathrm{NN}$ coupling constant is

$$
f_{\rho}^{2}=\frac{g_{\rho}^{2}}{4 M^{2}} m_{\rho}^{2}(1+\kappa)^{2} .
$$

The values of $g_{\rho}$ and $\kappa$ are not very well determined. Different authors give values $g_{\rho}^{2} / 4 \pi=0.4-0.6$ and values for $\kappa$ ranging from 3.7 (vector dominance model) to 6.6 [ref. $\left.{ }^{13}\right)$ ]. In view of these large uncertainties it simplifies the discussion to fix $g_{p}$ and to vary $\kappa$ alone. We therefore chose the value

$$
g_{\rho}^{2} / 4 \pi=0.52,
$$

in all the following calculations.

For the $\rho \mathbf{N} \Delta$ coupling constant we use the prediction of the static quark model:

$$
f_{\rho}^{*}=\sqrt{\frac{72}{25}} f_{\rho} \approx 1.7 .
$$

The corresponding prediction for pion photoproduction via the $\Delta_{33}$ resonant intermediate state is in good agreement with dispersion theory calculations ${ }^{14}$ ) and hence (3.19) may be assumed to be more reliable than the similar quark model prediction for the $\pi \mathbf{N} \Delta$ coupling constant which is $30 \%$ too small ${ }^{8}$ ).

In (3.16) we use the static form for the $\rho$-meson propagator as the energy transfer is much smaller than the $\rho$-meson mass. The Fourier transform of (3.16) is

$$
V_{12}^{\rho}(r)=\frac{f_{\rho}^{*} f_{\rho}}{4 \pi}\left\{\frac{2}{3} \frac{\mathrm{e}^{-m_{\rho} r}}{r} S_{1} \cdot \sigma_{2}-\frac{1}{3}\left(1+\frac{3}{m_{\rho} r}+\frac{3}{m_{\rho}^{2} r^{2}}\right) \frac{\mathrm{e}^{-m_{\rho} r}}{r} S_{12}^{*}(r)\right\} T_{1} \cdot \tau_{2} \text {. }
$$

Note that the sign of the tensor term in (3.20) is opposite to that of the pion exchange interaction in (3.13). Hence the exchange of the $\rho$-meson will act to cut off the pion exchange contribution. This has important consequences for the calculation of the pion absorption cross section, as already noted in ref. ${ }^{8}$ ).

The combination of pion and $\rho$-meson rescattering following isobar excitation is then summarized in the two-body operator

$$
M_{\Delta}^{+}(k, r)=\frac{f^{*}}{\mu} \frac{1}{\omega_{R}-\omega-\frac{1}{2} i \Gamma_{\Delta}}\left\{e^{\frac{1}{t i k} \cdot r}\left[V_{12}^{\pi}(r)+V_{12}^{\rho}(r)\right]\left(S_{1}^{+} \cdot k\right) T_{+2}^{+}+e^{-t \mid k \cdot r}(1 \leftrightarrow 2)\right\} .
$$

For the evaluation of the matrix elements of this operator we refer to appendix $B$.

\subsection{THE EFFECT OF FORM FACTORS}

We have hitherto described the pion and $\rho$-meson couplings to the baryons by zero range interactions. Vertex renormalization mechanisms will tend to spread the vertices over finite regions of space. This vertex structure is usually described by form factors. Since we are dealing with virtual mesons but baryons which are not 
far off-shell the form factors are functions only of the squared four-momentum of the meson. To take into account such form factors we replace the pion exchange potential in (3.6) by the expression

$$
V_{12}^{\mathrm{x}}\left(q^{2}\right)=\frac{1}{\mu^{2}} f^{*}\left(q^{2}\right) f\left(q^{2}\right) \frac{S_{1} \cdot q \sigma_{2} \cdot q}{q^{2}-\mu^{2}} T_{1} \cdot \tau_{2},
$$

with $q^{2}=q_{0}^{2}-q^{2}$. Here $f^{*}$ and $f$ are form factors associated with the $\pi \mathrm{N} \Delta$ and $\pi \mathrm{NN}$ vertices. We parametrize these functions by the monopole forms

$$
f\left(q^{2}\right)=f \frac{\Lambda_{\pi}^{2}-\mu^{2}}{\Lambda_{z}^{2}-q^{2}}, \quad f^{*}\left(q^{2}\right)=f^{*} \frac{\Lambda_{z}^{* 2}-\mu^{2}}{\Lambda_{z}^{* 2}-q^{2}},
$$

which are normalized to give the correct coupling constants for on-shell mesons. With these forms one may write the corresponding configuration space potentials as sums of Yukawa terms by use of a partial fraction separation.

Various values for $\Lambda_{x}$ and $\Lambda_{x}^{*}$ have been used in the literature, ranging from a few pion masses up to more than $1.5 \mathrm{GeV} / \mathrm{c}^{2}$. Theoretical estimates indicate that $\Lambda_{\mathrm{z}}$ cannot be much smaller than $1.0-1.2 \mathrm{GeV} / \mathrm{c}^{2}$ and $\dot{\Lambda}_{*}^{*}$ is probably of the same order of magnitude ${ }^{15}$ ). For simplicity we chose here $\Lambda_{z}^{*}=\Lambda \pi$.

In a similar way we include $\rho$-baryon form factors by the replacement

$$
f_{\rho}, f_{p}^{*} \rightarrow f_{\rho}\left(q^{2}\right), f_{p}^{*}\left(q^{2}\right),
$$

in eq. (3.16). For these form factors we use the monopole forms

$$
\frac{f_{\rho}\left(q^{2}\right)}{f_{p}}=\frac{f_{\rho}^{*}\left(q^{2}\right)}{f_{\rho}^{*}}=\frac{\Lambda_{\rho}^{2}-m_{\rho}^{2}}{\Lambda_{\rho}^{2}-q^{2}} .
$$

As the proper value for $\Lambda_{\rho}$ is not well known we perform calculations for a range of values for $A_{p}$ between 1 and $2 \mathrm{GeV} / \mathrm{c}^{2}$. The smallest natural scale for $A_{p}$ would be that given by the $\pi \omega$ vertex triangle diagram, i.e. something of the order of $1 \mathrm{GeV} / \mathrm{c}^{2}$, whereas the upper limit for $\Lambda_{\rho}$ must be $2 M$ which corresponds to the NN intermediate state in the general vertex diagram.

\subsection{NUMERICAL RESULTS}

We now turn to the discussion of the numerical results obtained with the model described above, which contains $\pi$ - and $\rho$-meson rescattering. In particular we investigate the dependence of the results on the $\rho$-meson coupling strength and the mass scale in the form factors.

In fig. 4 we essentially restate our earlier results ${ }^{8}$ ) for p-wave rescattering without form factors. The curves labelled IA are the impulse approximation results. The dashed curve is the result obtained with no final state interaction and the solid curve is the result obtained when the final state interaction is taken into account in the ${ }^{1} S_{0}$ and ${ }^{1} D_{2}$ final states. The curves labelled " $\pi$ " show the result when in addition to 


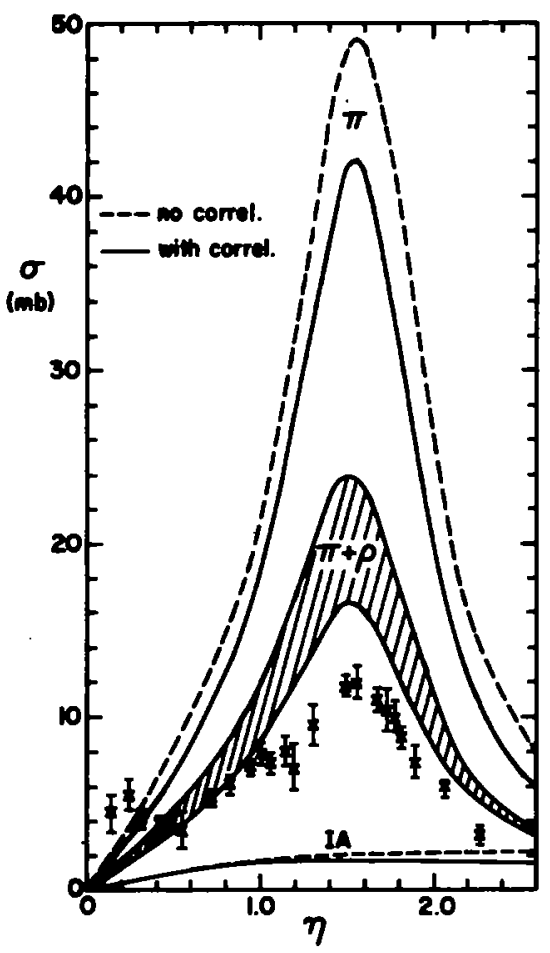

Fig. 4. The total cross section for P-wave pion absorption in $\pi^{+} d \rightarrow p p$, as a function of pion momentum $(\eta=k / \mu)$. The curves denoted "IA" represent the impulse approximation contribution, those denoted " $\pi$ " the result when pion rescattering is included and those marked " $\pi+\rho$ " when in addition $\rho$-rescattering is taken into account. The dashed curves are obtained without final state interactions in the singlet states. The shaded area for the $\pi+\rho$ results gives the variation of the cross section when $\boldsymbol{x}$ is changed between 3.7 (upper edge) and 6.6 (lower edge). The empirical values are from ref. ${ }^{1}$ ).

single nucleon absorption pion rescattering through the $\Delta_{33}$ resonance is included. Again the dashed line is the result obtained without final state interactions.

The final curves, those labelled " $\pi+\rho$ ", show the dramatic reduction of the cross section caused by inclusion of the $\rho$-meson rescattering mechanism. The shaded area in fig. 4 is limited by the values for $\kappa=3.7$ (upper edge) and $\kappa=6.6$ (lower edge), with $g_{\rho}$ kept constant at the value 3.18 . The results in ref. ${ }^{8}$ ) were obtained with $g_{\rho}^{2} / 4 \pi=0.65^{\dagger}$ and $\kappa=6.6$.

In fig. 5 we display the contributions from different partial waves in the final state to the lowest $\pi+\rho$ curve $(\kappa=6.6)$ of fig. 4. The final ${ }^{1} D_{2}$ wave is most important and gives rise to almost the whole cross section below the resonance region. In the region of the resonance triplet $\mathbf{P}$-waves are also important while the singlet-S and triplet-F waves are completely unimportant. (The ${ }^{3} \mathrm{~F}$ contributions have only been evaluated in the impulse approximation.)

t The value for $\sigma^{2} / 4 \pi$ was erroneouly given as 2.6 in ref. "). 


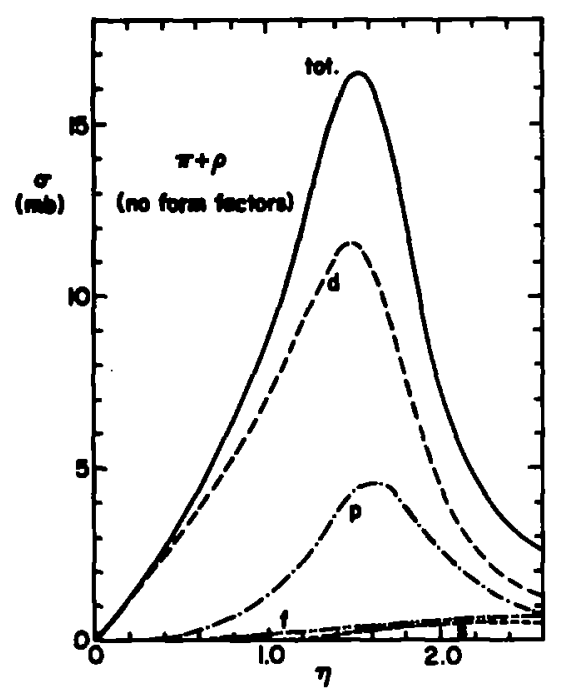

Fig. 5. The partial cross sections corresponding to the " $\pi+\rho$ " curve with $k=6.6$ in fig. 4 . The symbols s, p, $d$ and $f$ represent the lowest four partial waves in the final state.

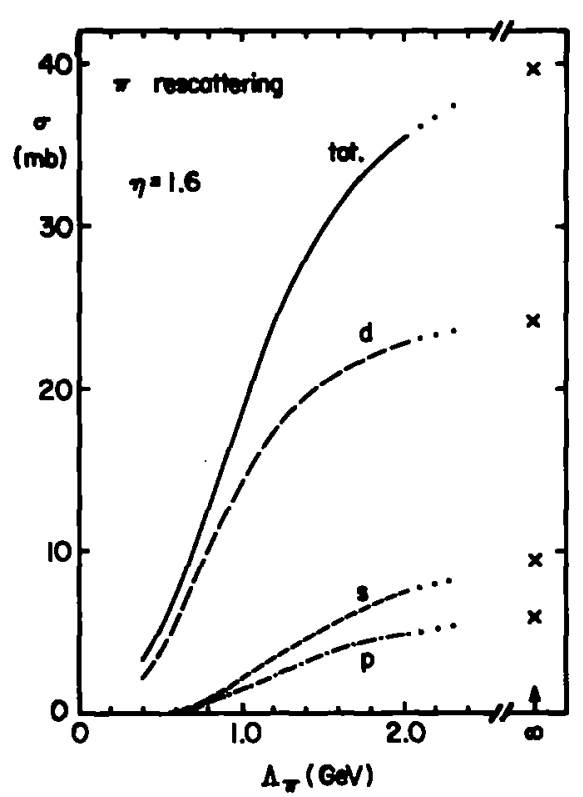

Fig. 6. The total and partial cross sections at the peak momentum $(\eta=1.6)$ for $\mathrm{IA}+$ pion rescattering as a function of the form factor mass scale $\Lambda_{a}$. The crosses at the r.h.s. represent the results for $\Lambda_{n}=\infty$.

The effect of introducing form factors of the type in eq. (3.23) on the pion rescattering contribution to the cross section is demonstrated in fig. 6 where we have plotted the peak value of the cross section, calculated at $\eta=1.6$, as a function of the parameter $A_{x}$. The model employed is that involving pion but not $\rho$-meson rescattering which for $\Lambda_{\pi}=\infty$ corresponds to the solid line labelled " $\pi$ " in fig. 4. The singlet $S$ and $D$ and the triplet $\mathbf{P}$ contributions are also shown separately. We note that the correct peak value for the cross section would be obtained with $\Lambda_{x}=800 \mathrm{MeV} / \mathrm{c}^{2}$ in rough agreement with the result of Gibbs, Gibson and Stephenson ${ }^{7}$ ) (their value of $600 \mathrm{MeV} / \mathrm{c}^{2}$ follows when S-wave absorption is included as well). The crosses on the right hand side of fig. 6 are the cross-section values obtained with no form factor $\left(\Lambda_{x}=\infty\right)$.

One should not interpret these results to indicate that the $\rho$-meson rescattering effect is simply equivalent to that of a form factor. The $\rho$-meson exchange mechanism counteracts the pion exchange mechanism only in the tensor part of the interaction, whereas in the spin-spin part it enhances the effect of the pion exchange mechanism. Thus, replacing the $\rho$-meson rescattering effect with a long range pion-nucleon vertex form factor would lead to too large partial cross sections to the singlet $S$ and too small partial cross sections to the triplet $\mathbf{P}$ waves. We conjecture that such deficiencies should be seen in angular distributions.

In fig. 7 we illustrate the effect of modifying both the pion and $\rho$-meson rescattering 


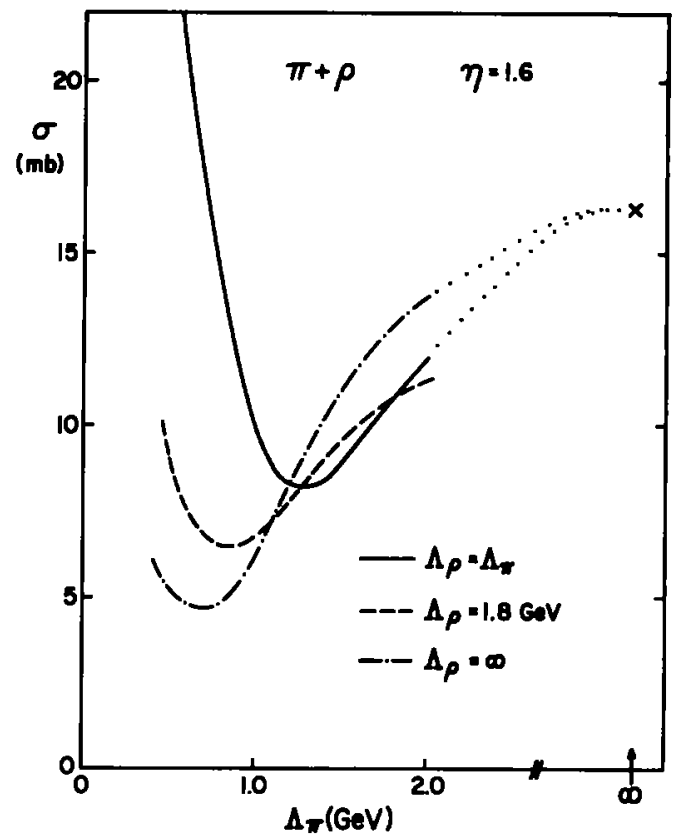

Fig. 7. The peak value of the total cross section as in fig. 6 for $\mathrm{LA}+\pi+\rho$ meson rescattering $(\kappa=6.6)$

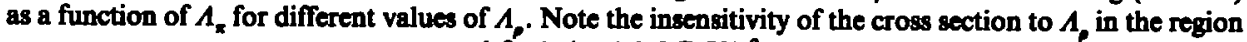
$1.2 \leqq \Lambda_{x} \leqq 1.6 \mathrm{GeV} / c^{2}$.

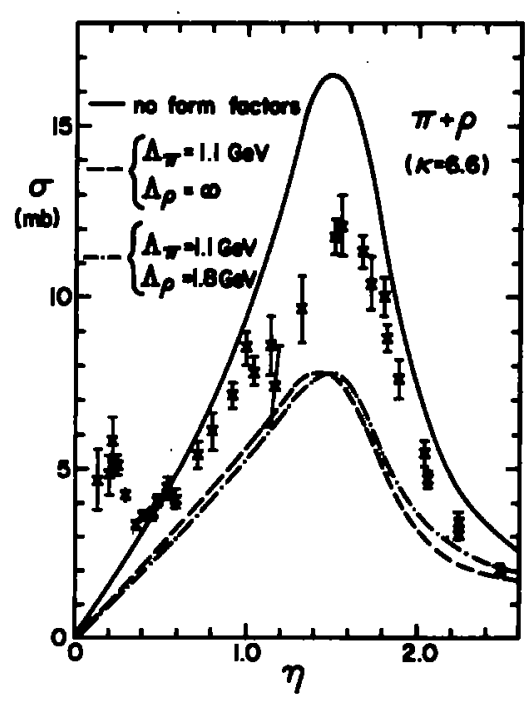

Fig. 8. The total $\pi^{+} d \rightarrow$ pp cross section for IA + $\pi+\rho$ rescattering $(x=6.6)$ plotted for different values of the form factor mass scales. The data are from ref. ${ }^{1}$ )

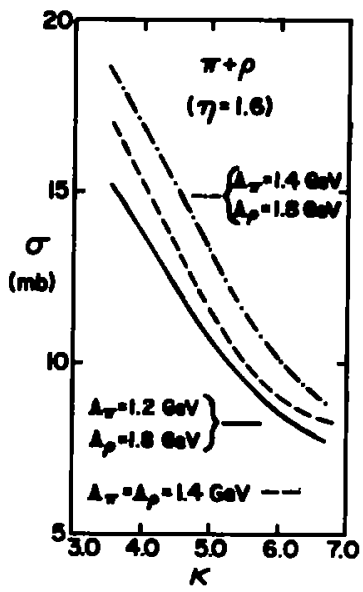

Fig. 9. The peak value of the total cross section for $1 A+\pi+\rho$ rescattering as a function of the $\rho$ tensor coupling parameter $x$. 
mechanisms by form factors as discussed in the previous subsection. We show the cross section at the peak momentum $\eta=1.6$ using the same model as was used in the results in fig. 5 above, but now modified by form factors. It is seen that unless the range of the pionic form factor is very long $\left(\Lambda_{\mathrm{z}}<1 \mathrm{GeV} / \mathrm{c}^{2}\right)$ the dependence of the results on the value of $\Lambda_{p}$ is not very strong. Once $\Lambda_{\mathrm{z}}$ is taken to be in the reasonable range 1.1-1.4 $\mathrm{GeV} / \mathrm{c}^{2}$ changing $\Lambda_{\rho}$ from 1.5 to $2.0 \mathrm{GeV} / \mathrm{c}^{2}$ changes the cross-section result by less than $10 \%$. The most important form factor effect is thus that associated with pion rescattering. The introduction of the pion form factor leads to a considerable reduction of the cross section. This is illustrated in fig. 8, where the total cross section has been plotted against incident pion momentum. That the cross sections obtained with the choice $A_{x}=1.1 \mathrm{GeV} / \mathrm{c}^{2}$ are some $30 \%$ too small as compared to the experimental values may be taken to be a strong indication against long range pion-nucleon form factors.

The most important parameters in the present model are $\Lambda_{x}$ and the $\rho$-meson tensor coupling constant $\kappa$. With the relatively large value 6.6 for $\kappa$ used in the results in figs. 7 and 8 one would need $A_{x} \geqq 1.5 \mathrm{GeV} / c^{2}$ in order to achieve agreement with the data. A smaller value for $\boldsymbol{k}$ would admit somewhat smaller values for $\Lambda_{\mathrm{x}}$ as there would then be less cancellation between the $\pi$ - and $\rho$-meson tensor interactions.

In fig. 9 we show how the peak value of the cross section at $\eta=1.6$ depends on the choice of $\kappa$. The relation between the choice of the values for $\Lambda_{x}$ and $\kappa$ is here rather obvious.

It is worth reemphasizing that in all the results presented in this section the single nucleon absorption amplitude (IA) was added to the rescattering amplitudes. Despite the smallness of the IA cross section the IA amplitude is important to include as it adds coherently to the rescattering amplitudes and leads to a large constructive interference term. If the impulse approximation amplitude were dropped without justification the cross-section results in figs. 5-9 would be reduced by almost a factor of 2.

The final state interactions have been taken into account above only in the singlet $S$ and $D$ waves. To obtain a rough estimate of their importance for the ${ }^{3} \mathrm{P}$ contribution to the cross section, we have studied the sensitivity of that partial cross section to a short range cut-off radius in the radial matrix elements for ${ }^{3} \mathrm{P}$ waves. It was found that changing that cut-off radius between zero and the unrealistically large value $0.7 \mathrm{fm}$ lead to changes of at most $10 \%$ in the partial cross section. Since this partial cross section contributes only a third part of the total cross section we conclude that the final state correlations in the ${ }^{3} \mathrm{P}$ waves may be safely neglected.

\section{S-wave abeorption mechanlums}

The P-wave rescattering mechanisms discušsed in sect. 3 provide a reasonable description of the pion absorption process in the region of the $\Delta_{33}$ resonance. To 


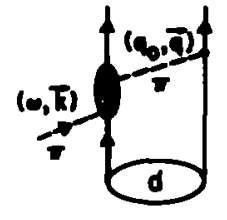

Fig. 10. Pion absorption through S-wave rescattering.

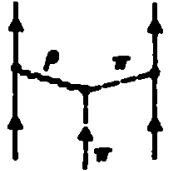

(a)

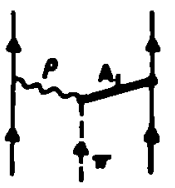

(b)

Fig. 11. (a) The S-wave rescattering caused by $\rho-$ exchange in the $\pi \mathrm{N}$ interaction. (b) Pion absorption by $\rho+A_{1}$ exchange.

account for the behaviour of the cross section near threshold S-wave rescattering mechanisms have to be included in the description as well ${ }^{3}$ ).

We describe S-wave rescattering, illustrated in fig. 10 , by the two-body operator

$$
\hat{M}_{\mathrm{s}}\left(q_{0}, q, k\right)=\frac{f}{\mu} \frac{\sigma_{2} \cdot q \tau_{2 \lambda}}{q_{0}^{2}-q^{2}-\mu^{2}}\left(\pi^{\lambda}(q)\left|F_{\mathrm{s}}(1)\right| \pi^{+}(k)\right) .
$$

This expression contains the half-shell S-wave $\pi \mathrm{N}$ scattering amplitude with an onshell incoming $\pi^{+}$of momentum $k$ and an off-shell intermediate pion with space-like four-momentum $\left(q_{0}, q\right)$.

The main contribution to S-wave $\pi \mathrm{N}$ scattering is due to $\rho$-meson exchange in the $t$-channel $\left.{ }^{16}\right)$. The isospin- 0 two-pion exchange contribution cancels almost completely against that of the nucleon-antinucleon intermediate state. Since it would be a complicated task to construct a dynamical model which maintains this cancellation for off-shell pions, we prefer to describe all the S-wave $\pi N$ interactions by the phenomenological zero-range Lagrangian used by Koltun and Reitan ${ }^{3}$ ):

$$
\mathscr{L}=-4 \pi \frac{\lambda_{1}}{\mu} \Psi \phi \cdot \phi \psi-4 \pi \frac{\lambda_{2}}{\mu^{2}} \Psi_{\tau} \cdot \phi \times \pi \psi .
$$

Here $\psi$ and $\phi$ are the nucleon and pion field operators and $\pi$ is the momentum operator canonically conjugate to $\phi$.

The second term in (4.2), when used to describe pion rescattering, corresponds to the diagram in fig. 11a in the limit of large $\rho$-meson mass. The first term describes isospin- 0 exchanges in the $t$-channel in the zero-range approximation.

The two coupling constants $\lambda_{1}$ and $\lambda_{2}$ are given in terms of the S-wave $\pi N$ scattering lengths as

$$
\lambda_{1}=-\frac{1}{6} \mu \frac{m+\mu}{\mu}\left(a_{1}+2 a_{3}\right), \quad \lambda_{2}=\frac{1}{6} \mu \frac{m+\mu}{\mu}\left(a_{1}-a_{3}\right) .
$$


With the values for the scattering lengths $a$ given by Bugg et al. ${ }^{17}$ ) we obtain from (4.3) $\lambda_{1}=0.003$ and $\lambda_{2}=0.05$.

With these values for the coefficients $\lambda$ the phenomenological Lagrangian (4.3) gives an adequate description of the $S_{31} \pi \mathrm{N}$ phase shift up to $300 \mathrm{MeV}$ pion lab kinetic energy, but leads to an overestimate of the $S_{11}$ phase shift above $50 \mathrm{MeV}$. As the S-wave rescattering mechanism is not very important far above threshold, this deficiency in the $S_{11}$ wave can be disregarded in the present context. Naturally the deficiency could be reduced by allowing if need be some energy dependence for the coupling strengths $\lambda$.

Using (4.2) to describe the rescattering vertex the amplitude (4.1) takes the explicit form

$$
\begin{aligned}
\hat{M}_{s}(k, r)=-i \frac{f}{\mu}\left(1+\frac{1}{\mu r}\right) \frac{\mathrm{e}^{-\mu r}}{r} & \left\{\mathrm{e}^{t i k \cdot r}\left(\sigma_{2} \cdot p\right) \sqrt{2}\left[2 \lambda_{1} \tau_{2+}-i \lambda_{2} \frac{\omega}{\mu}\left(\tau_{1} \times \tau_{2}\right)_{+}\right]\right. \\
& \left.-\mathrm{e}^{-t i k \cdot r}\left(\sigma_{1} \cdot p\right) \sqrt{2}\left[2 \lambda_{1} \tau_{1+}+i \lambda_{2} \frac{\omega}{\mu}\left(\tau_{1} \times \tau_{2}\right)_{+}\right]\right\} .
\end{aligned}
$$

To take into account the off-shell nature of the rescattered pion we shall multiply this expression with the same form factor that was introduced in subsect. 3.4 for the P-wave rescattering mechanisms.

In fig. 12 we present the results for the total pion absorption cross section obtained when the S-wave rescattering amplitude $\hat{M}_{\mathrm{s}}$ in (4.4) is included along with the $\mathrm{P}$-wave amplitudes $\hat{M}_{\mathrm{IA}}$ and $\hat{M}_{A}$ discussed in sect. 3 (the detailed expressions are given in appendix B). As expected the S-wave rescattering mechanisms contribute signifi-

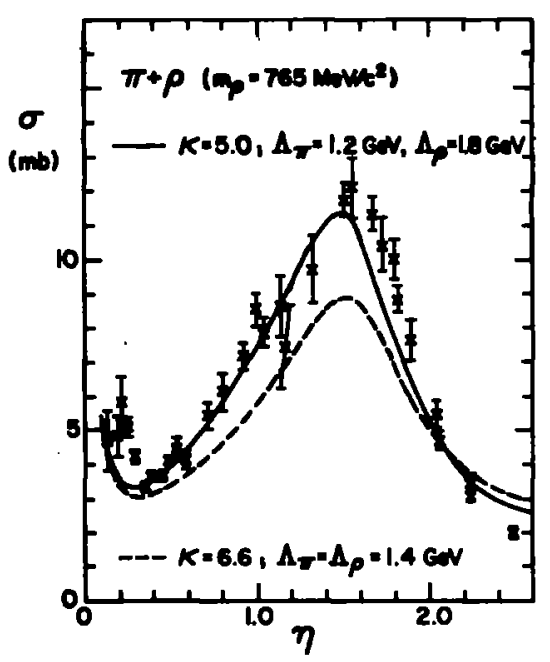

Fig. 12. Total cross section for $\pi^{+} d \rightarrow$ pp as in fig. 8, but including $S$-wave pion rescattering. Two choices have been made for the $\rho$-coupling $x$ and the cut-off parameters $A_{x}$ and $A_{p}$. 
cantly only near threshold ( $\eta \leqq 0.6$ ). The interference there with the other amplitudes is destructive. In the resonance region $(\eta \geqq 0.8)$ the inclusion of $\vec{M}_{s}$ changes the cross section by less than a mb.

In the results in fig. 12 we have used two different values for the $\rho$-meson tensor coupling strength. With the smaller value for $\kappa(5.0)$ a pion form factor with $\Lambda_{\mathrm{x}}=1.2 \mathrm{GeV} / \mathrm{c}^{2}$ reproduces the experimental cross section very well. With the larger value for $\kappa$ (6.6) agreement with the experimental curve would require $\Lambda_{\mathrm{x}}=1.6 \mathrm{GeV} / \mathrm{c}^{2}$. The results are rather insensitive to the $\rho$-meson form factor parameter $\Lambda_{p}$ as mentioned in the previous section.

Before leaving the topic of S-wave rescattering we point out that the impulse approximation would lead to a large contribution to S-wave absorption if in the correction term in the $\pi \mathrm{NN}$ Lagrangian (3.1) $E_{l}^{\prime}-E_{l}$ is interpreted as the energy of the incoming pion. We disfavour such an interpretation however as the quantities $E$ are defined in terms of nucleon momenta, and the lowest order approximation for $E_{i}^{\prime}-E_{i}$ is

$$
E_{i}^{\prime}-E_{i} \approx \frac{p_{i}^{\prime 2}}{2 M}-\frac{p_{i}^{2}}{2 M}=\frac{p_{i}+p_{i}^{\prime}}{2 M} \cdot q \neq \omega .
$$

Because of the explicit dependence on $q$ in the expansion (4.5) the correction term will appear only as a small relativistic addition of order $M^{-2}$ to the main $\sigma \cdot q$ term in the Lagrangian (3.1).

One might also ask whether not $\rho$-meson rescattering would give rise to an important effect for S-wave absorption as well. Such a mechanism could be the $\mathrm{A}_{1}-\rho$ exchange diagram in fig. $11 \mathrm{~b}$. As long as the sign of the $\pi \rho \mathrm{A}_{1}$ coupling constant is not known it is impossible to definitely assess the importance of this effect. Yet as these rescattering effects will not have the same tensor character as the P-wave rescattering mechanisms considered in sect. 3 we believe that the short range of the $\rho-A_{1}$ exchange interaction will make this effect rather unimportant.

\section{The p-meson mass distribution}

In sect. 3 the $\rho$-meson exchange mechanism was treated within the sharp resonance approximation. The large width of the $\rho$-meson calls for an investigation into the validity of this approximation and a treatment in which the $\rho$-meson is described as an interacting two-pion state. In fact the large effect of the $\rho$-exchange mechanism on P-wave pion absorption discussed in sect. 3 turns out to depend rather strongly on the mass of the $\rho$-meson. Thus there is strong reason to deal as well as possible with the $\rho$-meson mass distribution.

To construct a more realistic model for $\rho$-meson exchange we note that the isospin-1 two-pion-exchange nucleon-nucleon interaction can to a good approximation be written in the form ${ }^{18,19}$ )

$$
V_{p}(q)=\int_{4 \mu^{2}}^{\infty} \mathrm{d} t \rho(t) \frac{1}{3}\left\{\frac{2 t}{t+q^{2}} \sigma_{1} \cdot \sigma_{2}+\frac{q^{2}}{q^{2}+t} S_{12}(q)\right\} \tau_{1} \cdot \tau_{2} .
$$


Here $S_{12}$ is the usual tensor operator and the spectral function $\rho$ is

$$
\rho(t)=\frac{3\left(t-4 \mu^{2}\right)^{\frac{3}{2}}}{32 M^{2} \sqrt{ } t}\left|f_{-}^{1}(t)\right|^{2} .
$$

In (5.2) $f_{-}^{1}$ is the helicity amplitude for the reaction $\mathrm{NN} \rightarrow \pi \pi$ in the angular momentum 1 state $^{20}$ ).

To discuss $\rho$-exchange apart from the exchange of uncorrelated pions, we shall subtract the Born term (nucleon intermediate states) from $f_{-}^{1}$ in the following. This removal leads to the additional advantage that no double counting of wave function effects can arise.

The usual $\rho$-meson exchange potential is obtained from (5.1) in the zero width limit:

$$
\rho(t) \rightarrow \frac{f_{\rho}^{2}}{m_{\rho}^{2}} \delta\left(t-m_{\rho}^{2}\right)
$$

We now make the rather strong assumption that the $\rho$-meson exchange N $\Delta-\mathrm{NN}$ transition potential (3.20) may be interpreted as the zero width limit of an expression similar to (5.1) with a mass distribution $\rho^{*}$ that differs from $\rho$ in (5.2) only by the quark model scaling factor:

$$
\rho^{*}(t)=\sqrt{\frac{72}{25}} \rho(t)
$$

Instead of (3.20) we then have the generalized $\rho$-exchange transition potential

$$
V_{12}^{\rho}(r)=\frac{1}{4 \pi} \int_{4 \mu^{2}}^{\infty} \mathrm{d} t t \rho^{*}(t)\left\{\frac{2}{3} \frac{\mathrm{e}^{-r \sqrt{ } t}}{r} S_{1} \cdot \sigma_{2}-\frac{1}{3}\left(1+\frac{3}{r \sqrt{ } t}+\frac{3}{r^{2} t}\right) \frac{\mathrm{e}^{-r \sqrt{ } t}}{r} S_{12}^{*}\right\} T_{1} \cdot \tau_{2} \cdot
$$

In the numerical calculations we have fitted the squared non-Born part of the helicity amplitude by a Breit-Wigner form:

$$
\left|f_{-}^{1}(t)\right|^{2}=f_{0} \frac{\frac{1}{4} \Gamma^{2}}{\left(t-t_{0}\right)^{2}+\frac{1}{4} \Gamma^{2}} \quad\left(4 \mu^{2} \leqq t \leqq 50 \mu^{2}\right) .
$$

To the values for $f_{-}^{1}$ given by Höhler and Pietarinen (HP) ${ }^{13}$ ) eq. (5.6) gives an excellent fit with the parameter values

$$
f_{0}=3.0 \mu^{-4}, \quad t_{0}=29.5 \mu^{2}, \quad \Gamma=11.5 \mu^{2} .
$$

The corresponding amplitude model of Nielsen and Oades ${ }^{21}$ ) is not quite symmetric around the peak position $t_{0}$, but nevertheless a reasonable fit to that amplitude (squared) is obtained with the parameter values

$$
f_{0}=1.83 \mu^{-4}, \quad t_{0}=29 \mu^{2}, \quad \Gamma=11 \mu^{2} .
$$

The quadrature in eq. (5.5) was performed with Simpsons rule for those values of $r$ which are needed in the calculation of the radial matrix elements for the $\pi^{+} d \rightarrow p p$ amplitude. 


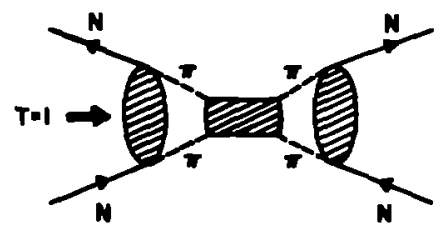

Fig. 13. Illustration of the isovector two-pion exchange interaction, where the $\rho$-meson is part of the interacting two-pion system.

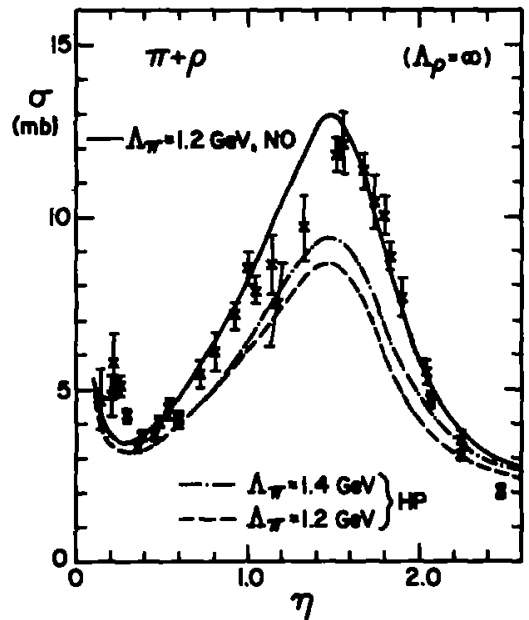

Fig. 14. Total cross section for $\pi^{+} d \rightarrow$ pp including S-wave pion and $P$-wave pion and $\rho$-meson is taken into account using the $\pi \pi \rightarrow \mathbf{N N}$ helicity amplitudes $f_{-}$(non-Born terms only) given by Nielsen and Oades ${ }^{21}$ ) (solid line, NO) and by Hohler and Pietarinen ${ }^{13}$ ) (dashed and dashed-dotted lines, HP).

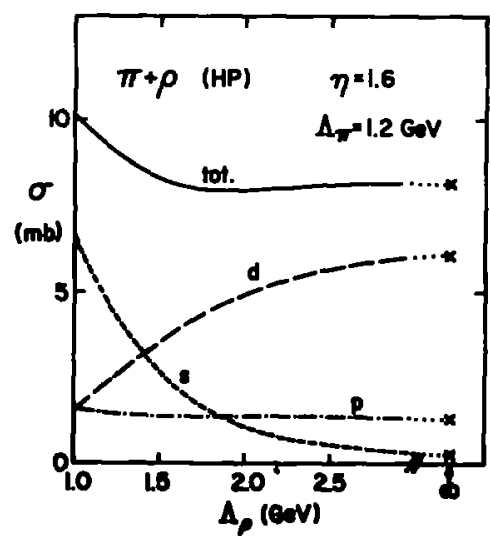

Fig. 15. Total and partial cross sections at $\eta=1.6$ for pion and $\rho$-meson rescattering a obtained with the Hobhler-Pietarinen helicity amplitudes as functions of the $\rho$-meson cut-off parameter $A_{p}\left(A_{p}=1.2 \mathrm{GeV}\right)$. The crosses

In fig. 14 we show the results for the total $\pi^{+} d \rightarrow$ pp cross section as obtained with these models for the $\rho$-meson mass distribution. With a value for the pion form factor parameter $\Lambda_{x}$ of $1.2 \mathrm{GeV} / \mathrm{c}^{2}$ the NO amplitude leads to a very good fit to the empirical cross section. With the HP amplitude the cross section comes out somewhat too low, by $20-30 \%$, if the value for $\Lambda_{\mathrm{x}}$ is taken to be in the range $1.2-1.4 \mathrm{GeV} / \mathrm{c}^{2}$.

The main difference between these two models for the $\rho$-meson mass distribution is to be found in their different effective $\rho$-meson coupling strengths. If we determine the effective $\rho$-meson coupling constants by comparison to the sharp resonance approximation (5.3) as

$$
\int_{4 \mu^{2}}^{50 \mu^{2}} \rho(t) \mathrm{d} t=\frac{f_{\text {eff }}^{2}}{m_{\rho}^{2}}=\frac{g_{\rho}^{2}}{4 M^{2}}\left(1+\kappa_{\text {eff }}\right)^{2},
$$


we find with $g_{\rho}^{2} / 4 \pi=0.52$ the values $\kappa_{\text {eff }}=6.8$ for the HP model and $\kappa_{\text {eff }}=4.95$ for the NO model. The value 6.8 for $\kappa_{\text {efr }}$ is in good agreement with the value 6.6 given by Höhler and Pietarinen ${ }^{13}$ ) as they used for $g_{\rho}^{2} / 4 \pi$ the value 0.55 . These results also indicate the propriety of our subtraction of the Born term from the helicity amplitude in order to separate $\rho$-meson exchange from uncorrelated two-pion exchange.

While the most important parts of the correlated two-pion exchange mechanism should be included in the description in terms of the helicity amplitude $f_{-}^{1}$ in (5.2), the effects of form factors at the pion-baryon vertices in fig. 13 are not. This is a consequence of the fact that the helicity amplitude describes the reaction $N N \rightarrow \pi \pi$ for on-shell pions, whereas in general the intermediate pions in fig. 13 are off-shell. There is no proper way to introduce the form factors within this framework short of a complete solution of the interacting two-pion two-nucleon system. One may though as an ad hoc substitute introduce form factors depending on the total momentum transfer variable $q^{2}$ in eq. (5.5) in the same way as was done for the sharp $\rho$-meson exchange interaction (3.16). Fortunately the results turn out to be rather insensitive to such an ad hoc $\rho$-meson form factor.

This is illustrated in fig. 15, where the total and partial cross sections for the lowest partial waves at the peak value $\eta=1.6$ are shown as functions of the $\rho$-meson form factor parameter $\Lambda_{\rho}$. The result obtained with $\Lambda_{p}=\infty$ corresponds to that in fig. 14 . The total cross section is remarkably constant for $\Lambda_{\rho} \geqq 1.5 \mathrm{GeV} / c^{2}$. It is interesting however that the partial cross sections are sensitive to $\Lambda_{\rho}$, but that there is a trade-off between the different components which leaves the total cross section insensitive.

\section{Conclusions}

The results presented above show that a rather nice explanation of the $\pi^{+} d \rightarrow$ pp total cross section is achieved once pion and $\rho$-meson rescattering is taken into account in addition to the single nucleon absorption amplitude (impulse approximation). The calculated cross section depends rathef strongly on two parameters: the $\rho$-meson tensor coupling constant $\alpha$ and the mass scale $\Lambda_{\mathrm{z}}$ of the pion-nucleon form factor. Because of the cancellation between the pion and $\rho$-meson rescattering effects we cannot fix these parameters independently: larger values for $\kappa$ allow smaller values for $\Lambda_{x}$ and vice versa (fig. 9).

As $\boldsymbol{\kappa}$ is known within certain bounds however, the present calculation requires similar bounds for $\boldsymbol{A}_{\boldsymbol{z}}$. In particular one cannot within the present framework obtain cross-section results in agreement with experiment with values for $A_{n}$ less than $1.2 \mathrm{GeV} / \mathrm{c}^{2}$ which would correspond to a long range form factor. We therefore have to interpret the use of long range form factors in refs. ${ }^{6.7}$ ) as a reflection of the omission of $\rho$-meson rescattering rather than as evidence for what the proper value for $\Lambda_{x}$ ought to be.

The present results turn out to be rather insensitive to the value for the mass scale 
$\Lambda_{\rho}$ of the $\rho$-nucleon form factor as long as $\Lambda_{\rho} \geqq 1.5 \mathrm{GeV} / \mathrm{c}^{2}$. Smaller values for $\Lambda_{\rho}$ would be hard to explain dynamically anyway as most of the $\rho \mathrm{NN}$ vertex diagrams have rather large mass intermediate states, the only exception being the $\pi \omega$ intermediate state.

The description of the $\rho$-exchange mechanism as a correlated two-pion exchange mechanism does not lead to results much different from those obtained with the sharp resonance approximation for the $\rho$-meson. With the recent models ${ }^{13,21}$ ) for the non-Born part of the NN $\rightarrow \pi \pi$ helicity amplitude $f_{-}^{1}$ used for the description of the $\rho$-meson we found it possible to obtain good agreement with the empirical cross-section values. The very strong effective $\rho$-meson coupling inherent in the Höhler-Pietarinen model for $f_{-}^{1}\left[\right.$ ref. $\left.\left.^{13}\right)\right]$ allows a calculation without any pionnucleon form factor at all and would indeed require some additional damping of the $\rho$-contribution.

The most important partial cross section is that to the ${ }^{1} D_{2}$ state. In this state the final state interaction is rather unimportant because of the centrifugal barrier. The ${ }^{1} D_{2}$ dominance thus explains the relative unimportance of final state interactions in the present calculations.

In the region of the $\Delta_{33}$ resonance the results do not depend very much on the values for the S-wave $\pi \mathrm{N}$ interaction strengths $\lambda_{1}$ and $\lambda_{2}$. Only near threshold where S-wave rescattering is the dominant effect do the particular values for the $\lambda$ matter. The uncertainty in these values is determined by the uncertainty in the values for the $\mathrm{S}$-wave $\pi \mathrm{N}$ scattering lengths.

There is a considerable number of other possible rescattering mechanisms that we have not considered. One such would be rescattering of an $A_{1}$ meson following the excitation of the intermediate $\Delta_{33}$ resonance. The $A_{1}$ rescattering operator has no matrix elements for singlet final states because of the positive intrinsic parity. Thus one would not expect the $A_{1}$ rescattering effect to be very large as the matrix elements for the ${ }^{3} \mathrm{P}$ state will be reduced in relation to those for pion and $\rho$-meson rescattering because of the short range of $A_{1}$ exchange.

We have not considered multiple meson rescattering effects in this paper. Such mechanisms are very complicated to estimate, although a subset of such effects may be included in a coupled channel treatment of the nucleon and $\Delta$-resonance components in the wave function. Such a coupled channel calculation has recently been performed by Green, Niskanen and Hăkkinen ${ }^{22}$ ), who found results in qualitative agreement with ours ${ }^{8}$ ). The reason that the full coupled channel treatment does not lead to results much different from those presently given is to be found in the very small weight of the $\Delta$-components in the two-nucleon wave function which seems to make the first order perturbation theory approach sufficient. The small differences obtained with the coupled channel and first order perturbation theory calculations are totally masked by the uncertainties in many of the input parameters, as e.g. the $\rho$-meson coupling constant.

We finally emphasize that the ultimate test of the present $\pi+\rho$ rescattering model 
for $\pi^{+} d \rightarrow p p$ will come in studies of angular distributions which are much more sensitive to the details of the model than the total cross section.

One of us (M.B.) wishes to thank Prof. G. E. Brown and Drs. M. Dillig and J. M. Richard for stimulating discussions.

\section{Appendix A}

The Lorentz-invariant form for the $\pi \mathbf{N}$ interaction in the pseudoscalar form is

$$
\left\langle\mathbf{N}\left(p^{\prime}\right)|\mathscr{L}| \mathbf{N}(p) \pi^{+}(k)\right\rangle=i g \sqrt{2} \bar{u}\left(p^{\prime}\right) \gamma_{5} u(p) \text {. }
$$

Since we deal with nucleons which are not far off-shell we use the usual expressions for the nucleon spinors $u$ to obtain

$$
\bar{u}\left(p^{\prime}\right) \gamma_{s} u(p)=\sqrt{\frac{(E+M)\left(E^{\prime}+M\right)}{4 M^{2}}} \xi^{+}\left[\frac{\sigma \cdot p^{\prime}}{E^{\prime}+M}-\frac{\sigma \cdot p}{E+M}\right] \xi .
$$

Here $\xi$ is the Pauli spinor for the nucleon. In (A.2) the quantities $E$ and $E^{\prime}$ are determined in terms of the momenta $p$ and $p^{\prime}$ and should not be interpreted as energies. The distinction is important only off-shell.

By expansion of (A.2) to the lowest orders in $p^{\prime}+p$ and $p^{\prime}-p$ one finds the result

$$
\left\langle\mathrm{N}\left(p^{\prime}\right)|\mathscr{L}| \mathrm{N}(p) \pi^{+}(k)\right\rangle=i \frac{g}{2 M} \sigma \cdot\left\{\left(p^{\prime}-p\right)-\frac{E^{\prime}-E}{4 M}\left(p^{\prime}+p\right)\right\} .
$$

To this order one obtains the same result if the pseudovector form for the Lorentz invariant $\pi \mathbf{N}$ interaction is used.

\section{Appendix B}

DETAILED EXPRESSIONS FOR THE CROSS SECTION

\section{B.1. SINGLET FINAL STATES}

The scattering amplitude for the ${ }^{1} \mathrm{~S}_{0}$ and ${ }^{1} \mathrm{D}_{2}$ final states may be written in the operator form

$$
T_{\mathrm{a}}=-i \frac{g \sqrt{ } 2}{M}\left\{A_{\mathrm{I}}\left(\sigma^{1}-\sigma^{2}\right) \cdot k-i B_{\mathrm{I}}\left(\sigma^{1} \times \sigma^{2}\right) \cdot k+A_{\mathrm{II}}\left(\sigma^{1}-\sigma^{2}\right) \cdot T-i B_{\mathrm{NI}}\left(\sigma^{1} \times \sigma^{2}\right) \cdot T\right\}
$$

Here the vector operator $T$ is defined as

$$
\boldsymbol{T}=\mathbf{3}\left(\boldsymbol{\rho}^{\prime} \cdot \boldsymbol{R}\right) \boldsymbol{P}^{\prime}-\boldsymbol{E} .
$$

The scalar functions $A_{1}$ and $B_{1}$ may conveniently be separated into partial wave components as

$$
A_{1}=a_{10}+5 P_{2}\left(\phi^{\prime} \cdot R\right) a_{12}, \quad B_{1}=b_{10}+5 P_{2}\left(\phi^{\prime} \cdot k\right) b_{12} .
$$


The total cross section, which by eqs. (2.6) and (2.7) is obtained by calculating the spin traces and integrating over the directions of $p^{\prime}$, then takes the form $\sigma_{8}=\frac{p^{\prime} k g^{2}}{3 M}\left\{\left|a_{10}+b_{10}\right|^{2}+5\left|a_{10}+b_{12}\right|^{2}+2\left|A_{11}+B_{11}\right|^{2}+4 \operatorname{Re}\left[\left(a_{12}^{*}+b_{12}^{*}\right)\left(A_{11}+B_{11}\right)\right]\right\}$.

The initial deuteron wave function has the standard form

$$
\psi_{\mathrm{d}}(r)=\frac{1}{r \sqrt{4 \pi}}\left(u(r)+\sqrt{\frac{1}{8}} w(r) S_{12}(f)\right)
$$

and the singlet scattering wave function is

$$
\psi_{f}(r)=\frac{1}{p^{\prime}} \sum_{L}(2 L+1) i^{L} \mathrm{e}^{i \delta_{L L 0}} F_{L L 0}\left(p^{\prime} r\right) P_{L}\left(\hat{p}^{\prime} \cdot \hat{f}\right)
$$

With these wave functions we obtain the following contributions to the scalar amplitudes $A$ and $B$ from the processes considered in sects. 3 and 4:

(a) Impulse approximation:

$$
\begin{gathered}
a_{10}=h_{0}+2 k_{02}, \quad a_{12}=h_{2}, \quad b_{10}=2 k_{02}, \\
A_{\mathrm{II}}=B_{\mathrm{II}}=k_{20} .
\end{gathered}
$$

The overlap integrals are defined as

$$
\begin{gathered}
h_{L}\left(q, p^{\prime}\right)=\frac{1}{p^{\prime}} \mathrm{e}^{-i \delta_{L L 0}} \int \mathrm{d} r F_{L L 0}\left(p^{\prime} r\right) j_{L}\left(\frac{1}{2} k r\right) u(r), \\
k_{L l}\left(q, p^{\prime}\right)=\frac{1}{p^{\prime} \sqrt{ } 8} \mathrm{e}^{-i \delta_{L L 0}} \int \mathrm{d} r F_{L L 0}\left(p^{\prime} r\right) j_{l}\left(\frac{1}{2} k r\right) w(r) .
\end{gathered}
$$

(b) Pion rescattering $(\boldsymbol{P}$-wave $)$ :

$$
\begin{gathered}
a_{10}=\xi_{x}\left(2 H_{022}^{\pi}+3 K_{002}^{\pi}-3 K_{022}^{\pi}\right), \\
b_{10}=\xi_{x}\left(H_{022}^{\pi}+3 K_{002}^{\pi}-3 K_{022}^{\pi}\right), \\
a_{12}=3 \xi_{x} K_{222}^{\pi}, \quad b_{12}=3 \xi_{x} K_{222}^{\pi}, \\
A_{\mathrm{II}}=\frac{1}{2} \xi_{x}\left(2 H_{202}^{\pi}-3 K_{002}^{\pi}\right) \\
B_{11}=\frac{1}{2} \xi_{x}\left(H_{202}^{\pi}-3 K_{202}^{\pi}\right) .
\end{gathered}
$$

The radial integrals are defined as

$$
\begin{gathered}
H_{L l m}^{\pi}=\frac{1}{p^{\prime}} \mathrm{e}^{-i \delta_{L L 0}} \int \mathrm{d} r F_{L L 0}\left(p^{\prime} r\right) j_{j}\left(\frac{1}{2} k r\right) Y_{m}(\mu r) u(r) \\
K_{L m m}^{\pi}=\frac{1}{p^{\prime} \sqrt{ } 8} \mathrm{e}^{-i \delta_{L L 0}} \int \mathrm{d} r F_{L L 0}\left(p^{\prime} r\right) j_{j}\left(\frac{1}{2} k r\right) Y_{m}(\mu r) w(r) .
\end{gathered}
$$


Here the functions $Y$ are modified Bessel functions of fractional order:

$$
Y_{m}(x)=\sqrt{\frac{2}{\pi x}} K_{m+\frac{1}{y}}(x) .
$$

The strength function in (B.9) is

$$
\xi_{\pi}=\frac{8}{27} \frac{f^{* 2}}{4 \pi} \frac{\mu}{\omega_{\mathrm{R}}-\omega-i \frac{1}{2} \Gamma_{\Delta}} .
$$

(c) $\rho$-meson rescattering: The contributions to the scalar amplitudes $A$ and $B$ due to $\rho$-meson rescattering have the same form as those due to $\mathbf{P}$-wave pion rescattering for singlet final states. They are obtained by the substitutions

$$
\xi_{\pi} \rightarrow \xi_{\rho}=-\frac{16}{75} \frac{g_{\rho}^{2}}{4 \pi}(1+\kappa)^{2}\left(\frac{m_{\rho}}{M}\right)^{2} \frac{m_{\rho}}{\omega_{\mathrm{R}}-\omega-\frac{1}{2} i \Gamma_{\Delta}}
$$

in (B.12) and $\mu r \rightarrow m_{\rho} r$ in the argument of the functions $Y_{m}$ in (B.10).

(d) $S$-wave pion rescattering:

$$
\begin{gathered}
a_{10}=-\xi_{s}\left(H_{011}^{\pi}-2 K_{011}^{\pi}\right), \quad b_{10}=2 \xi_{s} K_{011}^{\pi}, \\
a_{12}=\xi_{s}\left(H_{231}^{\pi}-2 K_{231}^{\pi}\right), \quad b_{12}=-2 \xi_{s} K_{031}^{\pi}, \\
A_{11}=\xi_{s}\left(H_{211}^{\pi}+H_{231}^{\pi}-2 K_{211}^{\pi}-2 K_{231}^{\pi}\right), \\
B_{11}=+2 \xi_{s}\left(K_{211}^{\pi}+K_{231}^{\pi}\right) .
\end{gathered}
$$

Here the strength function $\xi_{\mathrm{s}}$ is defined as

$$
\xi_{\mathrm{s}}=2 \frac{\mu}{k}\left(\lambda_{1}+\lambda_{2} \frac{\omega}{\mu}\right)
$$

\section{B.2. TRIPLET FINAL STATES}

The amplitude for scattering to the ${ }^{3} \mathrm{P}$ final state has the following general form if the spin-orbit force is neglected:

$$
T_{t}=-\frac{i g \sqrt{2} \pi}{M}\left\{3 C\left(\hat{p}^{\prime} \cdot \hat{k}\right)\left(\sigma^{1}+\sigma^{2}\right) \cdot k+D k\left(\sigma^{1}+\sigma^{2}\right) \cdot \hat{p}^{\prime}+E k \Sigma \cdot \hat{p}^{\prime}\right\} .
$$

Here the vector operator $\Sigma$ is defined as

$$
\Sigma=i\left[\sigma^{1} \cdot \hat{k} \sigma^{2} \times \hat{k}+\sigma^{1} \times \hat{k} \sigma^{2} \cdot \boldsymbol{k}\right] .
$$

The expression for the total cross section in terms of the scalar amplitudes $C, D$ and $E$ is

$$
\sigma_{\mathrm{t}}=\frac{2 p^{\prime} k g^{2}}{3 M}\left\{3|C|^{2}+|D|^{2}+\frac{2}{3}|E|^{2}+2 \operatorname{Re}\left[C^{*} D\right]\right\}
$$


The uncorrelated scattering wave function for the triplet state is

$$
\psi_{r}(r)=\sum_{L}(2 L+1) i^{L} j_{L}\left(p^{\prime} r\right) P_{L}\left(\hat{p}^{\prime} \cdot p\right)
$$

We obtain the following contributions to the scalar amplitudes from the processes considered in sects. 3 and 4:

(a) Impulse approximation:

$$
C=h_{1}+\frac{1}{5} k_{11}+\frac{9}{5} k_{13}, \quad D=\frac{9}{5}\left(k_{11}-k_{13}\right), \quad E=\frac{9}{5}\left(k_{11}-k_{13}\right) .
$$

The radial integrals are defined as

$$
\begin{array}{r}
h_{L}\left(k, p^{\prime}\right)=\int \mathrm{d} r r j_{L}\left(p^{\prime} r\right) j_{L}\left(\frac{1}{2} k r\right) u(r), \\
k_{L l}\left(k, p^{\prime}\right)=\sqrt{\frac{1}{8}} \int \mathrm{d} r r j_{L}\left(p^{\prime} r\right) j_{l}\left(\frac{1}{2} k r\right) w(r) .
\end{array}
$$

(b) $P$-wave pion rescattering:

$$
\begin{aligned}
& C=\xi_{x}\left(H_{110}^{\pi}+\frac{1}{5} K_{110}^{\pi}+3 K_{112}^{\pi}+\frac{1}{5} H_{112}^{\pi}+\frac{1}{10} K_{112}^{\pi}\right) \\
& D=\frac{9}{5} \xi_{x}\left(K_{110}^{\pi}+H_{112}^{\pi}+\frac{1}{2} K_{112}^{\pi}\right) \\
& E=\frac{9}{5} \xi_{x}\left(K_{110}^{\pi}+\frac{1}{6} H_{112}^{\pi}-\frac{7}{6} K_{112}^{\pi}\right)
\end{aligned}
$$

The radial integrals $H^{*}$ and $K^{\pi}$ are defined as in (B.10) with $F_{L L 0}\left(p^{\prime} r\right) / r p^{\prime}$ replaced by the uncorrelated wave function $j_{L}\left(p^{\prime} r\right)$. The strength function $\xi_{x}$ is given in (B.12).

(c) $\rho$-meson rescattering:

$$
\begin{aligned}
& C=\xi_{\rho}\left(2 H_{110}^{\rho}-3 K_{112}^{\rho}-\frac{1}{5} H_{112}^{\rho}+\frac{2}{5} K_{110}^{\rho}-\frac{1}{10} K_{112}^{\rho}\right), \\
& D=\frac{9}{5} \xi_{\rho}\left(2 K_{110}^{\rho}-H_{112}^{\rho}-\frac{1}{2} K_{112}^{\rho}\right), \\
& E=\frac{9}{3} \xi_{\rho}\left(2 K_{110}^{\rho}-\frac{1}{6} H_{112}^{\rho}+\frac{7}{6} K_{112}^{\rho}\right) .
\end{aligned}
$$

The strength function $\xi_{\rho}$ is defined in (B.13) and the structure functions $H^{\rho}$ and $K^{\rho}$ are defined as

$$
\begin{gathered}
H_{L l m}^{\rho}=\int \mathrm{d} r r j_{L}\left(p^{\prime} r\right) j_{t}\left(\frac{1}{2} k r\right) Y_{m}\left(m_{p} r\right) L(r), \\
K_{L=m}^{\rho}=\sqrt{\frac{1}{8}} \int \mathrm{d} r r j_{L}\left(p^{\prime} r\right) j_{l}\left(\frac{1}{2} k r\right) Y_{m}\left(m_{p} r\right) w(r) .
\end{gathered}
$$

(d) S-wave pion rescattering:

$$
\begin{aligned}
& C=\xi_{S}\left(H_{121}^{\pi}+2 K_{121}^{\pi}\right) \\
& D=-\xi_{S}\left(H_{101}^{\pi}+H_{121}^{\pi}+2 K_{101}^{\pi}+2 K_{121}^{\pi}\right)
\end{aligned}
$$


The strength function $\xi_{\mathrm{s}}$ is given in (B.15) and the structure functions $H^{\boldsymbol{x}}$ and $K^{\boldsymbol{x}}$ are obtained from (B.10) by the wave function substitution above.

\section{B.3. IMPULSE APPROXIMATION FOR ${ }^{3}$ F STATES}

If the ${ }^{3} \mathrm{~F}$ wave is included in the final state there is an additional term in the triplet amplitude (B.16):

$$
T_{i}^{\prime}=-i \frac{g \sqrt{2 \pi}}{M} F\left(\hat{p}^{\prime} \cdot \hat{k}\right) \Sigma_{p^{\prime}} \cdot \boldsymbol{k} .
$$

Here the vector operator $\Sigma_{p}$, is

$$
\Sigma_{p^{\prime}}=\sigma^{1} \times \hat{p}^{\prime} \sigma^{2} \cdot \hat{p}^{\prime}+\sigma^{2} \times \hat{p}^{\prime} \sigma^{1} \cdot \hat{p}^{\prime} .
$$

In order to obtain a simple expression for the cross section we replace the amplitude $C$ in (B.16) by

$$
3 C\left(\hat{p}^{\prime} \cdot \hat{k}\right) \rightarrow 3 C_{1} P_{1}\left(\hat{p}^{\prime} \cdot \hat{k}\right)+7 C_{3} P_{3}\left(\hat{p}^{\prime} \cdot \hat{k}\right),
$$

and the amplitude $D$ by

$$
D \rightarrow d_{0}+d_{1}\left(\hat{p}^{\prime} \cdot \hat{k}\right)^{2} .
$$

The expression for the cross section is then

$$
\begin{aligned}
\sigma_{\mathrm{t}}= & \frac{2 p^{\prime} k g^{2}}{3 M}\left\{3\left|C_{1}\right|^{2}+\left|d_{0}\right|^{2}+\frac{2}{3}|E|^{2}+2 \operatorname{Re}\left(c_{1}^{*} d_{0}\right)\right. \\
& \left.+7\left|c_{3}\right|^{2}+\frac{1}{5}\left|d_{2}\right|^{2}+\frac{2}{15}|F|^{2}+\frac{2}{3} \operatorname{Re}\left(d_{0}^{*} d_{1}\right)+\frac{6}{5} \operatorname{Re}\left(c_{1}^{*} d_{1}\right)+\frac{4}{5} \operatorname{Re}\left(c_{3}^{*} d_{1}\right)+\frac{4}{15} \operatorname{Re}\left(E^{*} F\right)\right\} .
\end{aligned}
$$

The impulse approximation contributions to the amplitudes $C, D, E$ and $F$ are, apart from those listed in (B.20) for the ${ }^{3} \mathrm{P}$ state,

$$
\begin{gathered}
c_{3}=h_{3}, \quad d_{0}=-\frac{9}{5} k_{31}, \quad d_{2}=9 k_{31}, \\
E=-\frac{9}{5} k_{31}, \quad F=-9 k_{31} .
\end{gathered}
$$

In (B.31) we only include those structure functions which involve the Bessel functions of lowest order.

\section{References}

1) C. Richard-Serre et al., Nucl. Phys. B20 (1970) 413

2) J. Spuller and D. F. Measday, Phys. Rev. D12 (1975) 3550

3) D. S. Koltun and A. S. Reitan, Phys. Rev. 141 (1960) 1413

4) S. Mandelstam, Proc. Roy. Soc. 244 (1958) 491

5) C. Lazard, J. I. Ballot and F. Becker, Nuovo Cim. 65B (1970) 1170 
6) B. Goplen, W. Gibbs and E. Lomon, Phys. Rev. Lett. 32 (1974) 1012

7) W. R. Gibbs, B. F. Gibson and G. J. Stephenson, in Meson nuclear physics - 1976, ed. P. D. Barnes et al., AIP Conf. Proc., vol. 33 (1976) p. 464

8) D. O. Riska, M. Brack and W. Weise, Phys. Lett. 61B (1976) 41

9) R. V. Reid, Ann. of Phys. 50 (1968) 411

10) H. Sugawara and F. V. Hippel, Phys. Rev. 172 (1968) 1764

11) G. F. Chew and F. E. Low, Phys. Rev. 101 (1956) 1570

12) G. E. Brown and W. Weise, Phys. Reports 22C (1975) 279

13) G. Hőhler and E. Pietarinen, Nucl. Phys. B9s (1975) 210

14) D. O. Riska and G. E. Brown, Phys. Lett. 33B (1972) 193

15) M. Brack and M. Dillig, to be published

16) S. Weinberg, Phys. Rev. Lett. 18 (1967) 188

17) D. V. Bugg, A. A. Carter and V. R. Carter, Phys. Lett. B44 (1973) 278

18) G. E. Brown, in Meson nuclear physics - 1976, ed. P. D. Barnes et al., AIP Conf. Proc., vol. 33 (1976) p. 655

19) M. Chemtob, J. W. Durso and D. O. Riska, Nucl. Phys. B38 (1972) 141

20) W. Frazer and J. R. Fulco, Phys. Rev. 117 (1960) 1603

21) H. Nielsen and G. C. Oades, Nucl. Phys. B49 (1972) 586

22) A. M. Green, J. A. Niskanen and S. Hakkinen, Phys. Lett. 61B (1976) 18 